6

The Culture of Humanities Research

\title{
Introduction
}

\section{Setting the scene}

This chapter covers a cluster of issues to do with the culture of humanities research. We found broad institutional and technological changes are affecting the way the humanities operate:

Internationalisation: there are more global networks, international collaborations and funding opportunities; increasingly, research is happening outside national and regional boundaries.

Interdisciplinarity: there is an established trend for major funding bodies in Europe and the US to insist that projects be interdisciplinary, involving the collaboration of scholars from different fields.

These phenomena are not new. Many researchers will say their fields have always been international and that interdisciplinary research has been on the agenda for decades. Nonetheless, because these developments are now quite established, there are questions about how they are affecting the culture of research. How are scholars reacting to them? Is there resistance? If so, what are the reasons and what forms does it take? While we conclude that interviewees broadly embraced the opportunities of global and interdisciplinary research, these come with challenges of language, power, finance and culture that are little understood, even within the research communities themselves.

We are not directly concerned with developments which might arise in the future and which might in turn affect or even transform the 
nature and culture of humanities research. For instance, many argue that traditional publishing methods, such as the scholarly monograph, will soon be outdated and that traditional hard copy journals will also disappear eventually. If such things do happen, they will change the nature of research. Similarly, the emergence of MOOCs as a form of teaching might challenge the traditional university model and, in turn, the way research is done. But to discuss these issues we would first need to gaze into a crystal ball to make appropriate predictions and then to conjecture their effects on academic culture. This is a perfectly valid exercise, but beyond the scope of this chapter. Instead, we begin with transformations that have already taken place and ask what reactions they are already producing.

As in other chapters, we make use of our interviewee's responses. The questionnaire included these topics and many of the responses were extremely detailed, giving us a rich source of information on which to draw. We shall also use reports and opinion pieces concerned with the issues.

\section{Attitudes to publication}

Before we go any further, we need to start with a fundamental feature of all humanities (and indeed any) research, publication. As we shall see with interdisciplinary research and internationalisation, current publication practices have a fundamental influence on the kinds of research carried out.

In our interviews we included a section on publication:

How do publications affect the way research is done, in particular through the assessment criteria they provide for hiring and promotion? What effects do the demands of getting published have on the work of younger researchers?

The answers to this question were wide-ranging but we should emphasise one very basic (and unsurprising) result. The majority of the respondents affirmed the importance of publication to hiring and promotion. A few, notably in India, suggested that the publication requirement is not as yet entrenched. But otherwise, the phrase 'publish or perish' would be an entirely apt way of describing the responses.

The majority of respondents also had some critical remarks to make about current publication regimes. The most common was that they privilege quantity over quality. Some respondents made the closely related point that the requirement for publication at regular intervals deters 
scholars from embarking on bigger projects, and so prevents longerterm planning and gestation. In reply to our question about younger scholars, many respondents (notably in Africa and the US) complained about the pressure put on scholars at the beginning of their careers. Another interesting theme that was occasionally detected was corruption in publication practices. Some respondents in Asia talked about people paying others to write papers for them, or using their influence and networks to get their name on a publication, even if they were not the authors. In Russia, some workshop participants said that, although nominally there was a system of peer review, in practice it was more or less non-existent. Very often, a paper was published because the author was able to pay the publisher.

This is just a glimpse of some of the views expressed in answer to our publication question. However, for the purposes of this chapter, we need to underline the great importance of publishing in humanities research for two reasons. First, current publication trends also favour the internationalisation of research, which may lead to a certain homogeneity. This was a concern noted by a number of respondents, and we is discussed in the internationalistion section of this chapter. Second, publication regimes may tend to favour monodisciplinary research, which creates a problem for those who wish to promote interdisciplinary work, given the sheer importance of publication for a scholar's career. We discuss this in the interdisciplinary research section.

\section{Internationalisation}

In the interviews we asked:

What effects is internationalisation (e.g. in recruitment patterns, institutional collaboration, networks) having on research and research activity?

This question drew a wide variety of responses, describing different ways internationalisation takes place and its different effects. Since we are surveying respondents' views on internationalisation, it may be helpful to recall the discussion of research themes and topics in Chapter 3. In the context of the current chapter it is interesting to ask whether a respondent mentioned themes that were limited to their own region or not. Out of 89 respondents, roughly one third mentioned only regional themes, while two thirds included international themes. The North American results were particularly striking since almost all were international in focus. One respondent (NA9) explicitly commented on the 
way research has become much more internationalised over the past few years. One interesting answer, which occurred a few times in quite different regions, is that internationalisation is not particularly new. Scholars have long been international in their choice of topics, in what they read and refer to, and in the conferences they attend.

What is new, however, is the growth of international networks, funding initiatives, publishing and ranking systems. According to many of our respondents, all this brings with it the risk of homogeneity. This, at least, is the view of around half of the Asians we interviewed, as well as half the Africans and half the non-Anglophone Europeans (excluding the Russians). Only one Anglophone scholar referred to the issue (an American working frequently in Asia). Although this does not constitute a majority it is still a very significant minority, not least because this same message is consistently voiced in quite different parts of the world.

In what follows we shall distinguish three different forms of internationalisation, which are thought to lead to homogeneity: networks, publishing and ranking.

\section{International networks}

Let us start with the increase of international research networks, collaboration and overseas funding. One respondent from Norway noted the phenomenon, but remained neutral about its effects:

E6: The main national funding body, the Norwegian Research Council, puts great stress on internationalisation when applications are evaluated. This means that projects which can show that they are part of international networks, have a greater chance of being funded, other things being equal.

But the following respondents were far from neutral. They all thought that such internationalisation leads to the Westernisation or Northernisation of research agendas, even though it has other benefits: ${ }^{1}$

ME1: In addition to EU projects ... there are many cooperation agreements between Jordan and Western universities, for both faculty and students. Since 9/11, Western countries are getting more interested in the Middle East, and a lot of people are coming to Jordan. Also, some agencies are working in Jordan, e.g. US agencies and the Japanese International Cooperation Agency (JICA). In most cases they determine the research topics and impose them on us 
(e.g. democracy, women's rights). So, although internationalisation is increasing, there's a lack of autonomy for us in the selection of research themes.

As4: Internationalisation of research and research activity brings in both competition and collaboration. Both are important factors for the generation of new findings. However, because of the increased competition from the non-native species, some research fields may not survive and may become extinct eventually. From the view point of preserving cultural heritage, it may be beneficial to preserve these endangered species.

ME2: At present our vision of internationalisation is still too Western, which makes it difficult to confront problems about the East, especially the Middle East.... As regards its effects, internationalisation pulls research in a Western direction, i.e. towards the EU and the US.

ME3: In general, the existence of an international market homogenises research. On the negative side, this leads to fewer surprises, but on the positive it means people communicate better.

Af3: a negative effect [of internationalisation] is the increasing dominance of research and HE agendas by northern universities. Universities from the North are increasingly becoming powerful and dominant in the running of research affairs of universities in the South. The danger of research imperialism is rife.

Af5: in poor countries like Tanzania research funds are almost exclusively externally sourced. Such funds often come with an agenda. So researchers may be forced to work on topics that may not have relevance to local communities.

Af6: it is not difficult to see how internationalisation leads to colonisation of disciplines/spaces when there are unequal structures, particularly in situations where the collaborations are tied to funding availed by collaborating institutions/partners in the West. Even in intra-Africa collaborations, I have found that some of the top-rated universities (e.g. UCT, Stellenbosch, etc.) have some departments that are basically colonial, structurally and conceptually, as evidenced by the courses taught there and, obviously, when one is involved in collaborations with colleagues from these spaces, the undertaking is not really one of mutual equality but one where a colonial department is striving to reproduce itself on (or drive the agenda of a relationship with) a poor and supposedly naive department in another African university, teaching courses that are structured/conceptualised within postcolonial approaches. 
Af13: Internationalisation has been crucial in shaping and improving disciplinary discourse, and in improving the pool of postgraduate students. Cutting edge research has taken place in contexts where researchers deliberately seek to encourage transnational collaboration. The danger though has been a process where (South) Africa is seen as a site of theoretical experimentation when theories emanating from the North are used indiscriminately and unmediated by local conditions. A form of intellectual mimicry is the result.

\section{Internationalisation in publishing}

It is not just internationalisation in the form of international networks that may lead to homogeneity, some respondents also pointed the finger at publication. The underlying concern could be expressed as the following argument: [a] publishing, especially journal articles, is key to professional advancement; $[\mathrm{b}]$ the more prestigious the outlet, the more useful the publication will be to a scholar's career; [c] but, typically, prestigious means an international and English language journal; [d] the sorts of themes (and criteria) appropriate to these journals tend be Western in focus. The upshot of [a]-[d] is that institutional conditions militate in favour of Anglophone homogeneity.

As1: Internationalisation is useful... But the English language has become the common language, and when it comes to research about a non-Anglophone culture, especially about Asian culture, there's an adverse effect, which results from the difficulty of expressing oneself in this common language. The standard of research has dropped and research has become isolated.

As10: Internationalisation is increasingly important, and this is not always for the best. As evidenced, for instance, by the pressure to publish in international journals. This forces me to choose Western topics and play by Western rules.

The European responses were interestingly mixed on the issue. Although some were more positive (as we shall see below), others saw the downsides of internationalisation in publishing:

E2: The need for internationalisation has a profound impact both with regard to the choice of research topics and the need to enhance our international visibility. At a practical level English is becoming the lingua franca in the humanities as well. The German 
competition for excellence required that all project applications had to be submitted in English.... There are many colleagues who insist, rightly so, that teaching and writing in their native tongues are indispensable to grasp the full meaning of their work.

E3: [Publications and career development] are affecting [research] very much now, though not in recent times. In Spain, there are fewer publishing houses than elsewhere in the EU (e.g. France). So, because of the small number of outlets, in the past there was less pressure to publish. But since 2000 there is more expectation from the EU for us to publish, especially in English. This forces us to play by foreign rules and risks undermining what is distinctive about our research (e.g. in Catalan).

E5: [How has publication changed the way that research is conducted?] Well that's very easy; it affects it a lot. In particular with my young researchers I can see it clearly and it affects them dramatically. There are two real effects: one, no national publications, everything is in English; two, no monographs but everything passed down in articles because that is what count in the publication assessment system. Then of course, this is a huge challenge to many traditions within the humanities itself, not to mention disciplines such as history, anthropology and even media studies for that matter.

E14: Internationalisation, when it allows comparison, is basically good. The problem with internationalisation is that it is more and more homogenous and it tends to be more of an Americanisation. This is worrying for sure. The fact that we have to publish in English rather than in our mother tongue, which everyone masters better than the other languages one may speak, is a big issue. It also affects cultural diversity, which is essential for the humanities, and important as well for sciences. If internationalisation is becoming too homogenous, there would be only one kind of network, and then one kind of research. We will lose our rich diversity.

Three African respondents drew the contrast between local and nonlocal outlets (two merely registered the phenomenon, one was openly critical):

Af1: This university has strict promotion criteria based on publications. Without this minimum number of publications, a person does not get promoted. [Does this specify types of publication: journal article/book; so-called 'international' versus national or regional journals etc?] Definitely. An international journal is interpreted as 
any journal published in the Americas, Europe and some parts of... Asia...

Af5: University policy on publication avenues:...for promotion, academic researchers are required to publish in world-recognised journals almost all of which are in foreign languages and for sure, not found in local circulations. Hence, whatever is published there does not reach the mass of Tanzanians. It is a pity! Given the chance, I would put a condition that one must publish in local journals and in a local language (say Kiswahili), along with the international avenues, in order to get promotion.

Af4: All researchers need avenues and outlets for publishing research results. This is even more urgent for younger researchers. Local journals are not always as viable as world-class journals and books which accord visibility and comparability. This is emphasised for promotion in my university.

\section{International ranking systems}

Another issue that concerns is the perception of international ranking systems. In a different section of our questionnaire, we asked:

What effects do ranking systems have on research, on the behaviour of researchers and on the management of research in your area? (Give examples to illustrate your answers.)

This question drew a wide variety of responses. Although we shall not analyse them here, we can say that over half the respondents were, in some way, sceptical of ranking systems (respondents from Asia and the US were the most negative). Some were sceptical in the sense of denying that ranking systems had any significant effect on research activity. Others thought that they did, but were sceptical about the alleged benefits of ranking. Some respondents were more positive and thought of international rankings as a means to challenge bad research practice in their region.

For the purposes of this chapter, the important point to draw out from our respondents' concerns how ranking systems tie up with internationalisation. Here are two extracts:

E7: What is demanded, that will be received. During the past five years the share of ISI and ETIS 1.1 articles (in journals with highest ranking according to our national assessment system) has remarkably increased in the humanities (from $5 \%$ of all 
publications in 2008 to $15 \%$ in 2011). At the same time, articles in domestic issues are steadily decreased in number (from $85 \%$ to $75 \%$, while the number of monographs has stayed on the same level, instead of increasing). As the monographs published by national publishers are not valuated as high as the articles in international journals, the researchers are not enthusiastic to write them.

R1: University ranking does affect research in my university since its global aim is to get higher in international rankings by means of stimulating publications in international journals that are included in bibliographic databases. That orientation stimulates publishing in English and disqualifies publications in Russian.

These comments are neutral and do not actually criticise the system, though they point to a link between ranking systems and publishing in international journals (with language implications). But three respondents (working in East Asia, Latin America and Africa) were explicitly concerned:

As14:...citation indices are mostly based in the United States and this is creating American hegemony and favouring contributions based on American views and research paradigms.... East Asia has its own distinctive cultures and its own ways of looking at and analysing things, and this is systematically diminished by the current ranking system.

LA4: International rankings have become an obsession of the university in the last few years... The downside is that many areas of research, like Brazilian political thought, that are very important locally, get underevaluated because they have low potential for international diffusion.

Af10: Rankings also have the tendency to ignore research reports in journals which do not originate from the global North. This depresses the development of publication outlets (within Ghana, and the region as a whole) that may have more regional and continental relevance.

The problem could be put as follows. The most prestigious rankings are international, and it is these that will attract the interest of academic managers. The danger is that the criteria of success tend to be set by Western universities, especially those in the US. So when an academic manager forces humanities researchers to 'chase' rankings, they are in 
effect asking them to make their research fit Western standards. Yet again, this has the effect of homogenising research.

\section{Redressing the balance}

In contrast, some respondents positively embraced internationalisation, whether in the context of networks, funding opportunities, publication or rankings. Some were unreservedly positive about the effects of internationalisation in general:

R2: I have no doubt that internationalisation is very good for the quality of the research.

E10: In many respects, internationalisation encourages research by accelerating the exchange of knowledge and methodologies between scholars of different affiliations. It is also good by favouring the creation of international research networks.

Af11: Internationalisation is the best practice in research activity. This is germane to getting quality delivery of knowledge and it is also important in knowledge transfer. There must be institutional collaboration and networks for proper knowledge transfer, especially as the world has become a global village.

Af12: Internationalisation is good for research. Indeed, institutions are actively encouraging efforts that promote internationalisation in terms of research collaboration and interdisciplinary work between researchers from collaborating institutions.

R4: I think that internationalisation has big influence, especially in Russia where scientific institutions in times of the USSR were ideologised. Their activity was complicated by all sorts of political and social obstacles: the restriction to hire Jews, non-conformists and other groups, difficulties in career development for the nonparty people, sometimes for women, etc. In modern Russia most of these complications are removed, but scientific institutions are weak and not well informed. Internationalisation is an important way of maintaining the intellectual potential of Russian science. Unfortunately, in Russia now the political elite thinks of internationalisation of science as something suspicious. The country's leaders in their public statements speak disapprovingly of those in Russia who receive foreign grants for research and advocacy work, allude to the fact that these people are not loyal to Russia and 'betraying' her for the sake of foreign interests. This 'demonisation' of those who receive grants may harm cooperation of Russian humanitarians with international scientific institutions. 
Two Europeans (from Sweden and the Netherlands) actually encouraged publication in English:

E1: If we take the language issue, then we should continue to publish more in the big languages and, of course, English is the biggest. If we think we have reached interesting results then we should be encouraged to spread these results as widely as possible. And I also think that precisely because people in the humanities are often subject to critique, we need to stick together globally.... There are many strong reasons for having a high international profile and therefore publishing in English is one way to go.

E4:... methodological nationalism is a reaction to globalisation....those who subscribe to methodological nationalism think that writing in English only allows you to approximate to the real thing in terms of national culture. So they assume the best work has to be... published in local rather than international journals. ${ }^{2}$

In general, it was interesting to see that not one of our Russian respondents (nine in all) had anything negative to say about internationalisation; ${ }^{3}$ either they were neutral, or they were positive and lamented the lack of it. Similarly, none of the Indian respondents mentioned any downside to internationalisation. ${ }^{4}$ One was particularly positive:

As3: Whatever internationalisation there has been for research collaborations and networks has been fruitful for research activity in Indian universities.

On the language issue, we should note that there is a report on the humanities in India (sponsored by the UK's AHRC, but written by the India Foundation for the Arts), which does criticise a bias towards the use of English, to the detriment of regional research, though of course the explanation for this reaches back to India's colonial past rather than present-day globalisation. ${ }^{5}$

\section{Conclusion}

Clearly this is an important issue over which scholars are divided. Our purpose is not to take sides, but to draw attention to the seriousness of the problem and recommend that international funding bodies take note, with a view to more extensive consultation. This is a particularly acute dilemma for the humanities. As some respondents note, it is of the nature of the humanities to work closely with the nuances of 
particular languages and cultures. So perhaps there is something in the claim that research can be endangered by increasing Anglophone homogeneity, in a way that STEM subjects are not. However, comparative research depends on a lingua franca between researchers. The warning against 'methodological nationalism' (E4) is particularly salient in this regard. At the same time, the humanities are especially at risk of running into conflict with their own governments, or at least suffering neglect, and hence they benefit hugely from international support and collaboration. This dilemma cannot be swept under the carpet.

\section{Interdisciplinary research}

\section{What is interdisciplinary research?}

In this section we make extensive use of the responses to Section 4 of the questionnaire:

Is your own research monodisciplinary or interdisciplinary?

What are the benefits or disadvantages of each type of research?

The overall response to the first question was quite striking: of 89 respondents, 63 were interdisciplinary, and only 13 were monodisciplinary. ${ }^{6}$ So far, so clear. But in the course of describing their research as interdisciplinary or otherwise, some respondents reflected explicitly on how to define the term and a number of others revealed their implicit assumptions about the nature of interdisciplinary research. This material is quite complex and we need to analyse it in order to make sense of their responses to the second question about the benefits or disadvantages of each type of research.

Looking at the results as a whole, we can make the following distinctions:

(A) Taking the term interdisciplinary to apply to an individual researcher, one could mean at least three things, presented here in order of strength:

\section{Communicating with other disciplines:}

NA5: My conception of interdisciplinary is that it is one person whose work is addressed to multiple communities.

E9: [Interdisciplinary research] could simply involve bringing two disciplines into conversation with each other. 
ME4: In my own work, I pay attention to language, not to describe the formal aspects of language as some linguists might, but to see how certain linguistic phenomena might be interpreted in a social context. I point out certain things that a historian or sociologist might not have noticed, but I don't start from a historical or sociological theory. So my own way of being interdisciplinary is to notice a difference in a linguistic term, but hand over to the historian to interpret the fact, to make something out of it. I don't attempt to become a historian or a sociologist, but my work complements theirs. Based on my particular expertise, I try to make people in other disciplines notice things.

2. Borrowing the methods, etc. of other disciplines:

R2: My own research is interdisciplinary as I use historical sources, methods and approaches, alongside with anthropological ones. E9: [Interdisciplinary research] might involve one discipline using the tools and vocabulary of another (something often frowned upon, as it may involve one discipline misunderstanding the tools of the other).

\section{Mastering other disciplines:}

NA3: True interdisciplinarity is so very hard to do. True interdisciplinarity would require a thorough knowledge of more than one discipline.

NA11: I think the word interdisciplinary is a misnomer. I think there is almost no good interdisciplinary research. I think there is good multidisciplinary research. You have to be strong in a discipline working with other people who are also strong in a discipline. Really interdisciplinary people are rare.

E1: I would say that my research is mainly monodisciplinary, it's comparative but it's not really interdisciplinary. As I said, you have to demand more than what I do in order for something to be really interdisciplinary.

As10: The disadvantages of interdisciplinary research are that it is difficult for one person to master more than one field.

(B) Taking interdisciplinarity to involve two or more researchers it could mean collaborative interdisciplinarity (multidisciplinarity):

NA6: A lot of my research is fairly interdisciplinary these days, though I have rather tough standards you have to meet in order 
to call yourself interdisciplinary. I think we're well beyond the era in which borrowing methods and discourse from other disciplines makes one interdisciplinary. The bar that I would set today is that you have to be working with other people in different disciplines on common projects.

NA10: Interdisciplinary work is almost by definition collaborative, because it's very hard for people to control knowledge within one discipline, much less many.

As8: Even in the single field of linguistics and culture, you may see a single scholar, or indeed scholars from a single country, with a different source of language and materials/resources that are oftentimes different. They have to work together and conduct collaborating research.

E13: I'd divide [interdisciplinarity] into two types: soft and hard.... Hard interdisciplinarity involves specialists from different disciplines gathering together over a common object or text. As a result, their own individual ways of operating have to change.

E2: Of course the term interdisciplinary is vague. Do we mean that scholars of different fields collaborate and that each brings in a specific expertise? Or do we mean, as some people claim, that we need to dissolve the disciplines altogether?

(C) Post-disciplinarity:

The last extract suggests a different concept altogether, also alluded to by another respondent:

E9: I've heard the phrase post-disciplinarity used. I'm not sure I understand it, but I don't think interdisciplinarity will or should take us to a point where disciplinary boundaries actually vanish.

We shall leave post-disciplinarity on one side, since it was only of marginal interest to our respondents. ${ }^{7}$ By contrast, collaborative interdisciplinarity is extremely important to the humanities, as we shall see in more detail below. For the purposes of this chapter we shall refer to it as 'multidisciplinarity'. One point should be uncontroversial: a multidisciplinary project will require individual researchers to be interdisciplinary in the first sense mentioned above (A)1, and to an extent the second (i.e. using the sources of other disciplines). But it surely does not require the third, (A)3, a strong sense of mastering another discipline; indeed the 
function of collaborative projects is precisely to avoid requiring individual researchers to be interdisciplinary in this way.

\section{The value of interdisciplinary research}

Next we turn to the value of interdisciplinary research. Many of our respondents claimed both advantages and disadvantages for it:

\section{Advantages}

1. Interdisciplinary research is broad and synoptic.

This point was made by seven respondents, Af1, Af6, As1, E4, E10 and:

Af3: Interdisciplinary approaches are more holistic, and so address issues more comprehensively.

NA2: It is essential that you simultaneously have specialists and that you have people trying to make sense of the bigger picture.

2. Many complex research questions simply require interdisciplinary research.

Here are the most explicit statements, though the point was made implicitly by many others: ${ }^{8}$

ME4: The problem of monodisciplinary work is that the humanities are essentially plural; there is a complementarity between humanities disciplines.

E5: The complexity of the empirical questions simply demands that more researchers from more diverse backgrounds come together to answer the question, that's one trend.

LA10: Monodisciplinary research is necessary for improving the technical tools of the trade in a specific field. Multidisciplinary research is necessary because almost all humanistic questions have to do with more than one discipline.

3. Interdisciplinary work is much more likely to throw up innovative methods and results, and to open up new horizons.

This point was made by 13 respondents, As4, As6, As9, As10, Au2, E15, ME3, ME6, NA 8, NA16, R5, R6, R9 and:

E12: The benefits of such work are huge, with each collaborator having the chance to discover not only new materials and insights, 
but whole new paradigms to bring to bear on the topics under discussion.

4. Interdisciplinary research facilitates translation by enabling humanities scholars to link up with researchers in other disciplines, e.g. medicine (resulting in bioethics), and through that discipline allow its own results to feed through to society at large.

This point was made by 8 respondents, Af11, As13, ME3, R6, NA8, R6 and:

E6: for the humanities as a field it is important to engage with other disciplines, both within the humanities and with fields outside our own. We need to establish and communicate to the outside world the importance of the humanities for the social and economic development of the society we are a part of, and we must encourage our own researchers to take up these challenges in their research.

Af9: Interdisciplinarity lends itself to application beyond mere publication. Monodisciplinarity [is] limited in results uptake...

ME4: We'll always need monodisciplinary research. And I don't use interdisciplinary work to find theoretical research questions, but to find applications.

5: Interdisciplinary research helps one better understand one's own discipline.

This point is worth highlighting. It was made by only three respondents, but they came from three very different countries (Canada, China and Romania), working in literature, history and philosophy:

As11: Interdisciplinary research is good when it arises from deep reflection on the boundaries of one's own discipline and on the limitations that these boundaries may impose.

E10: Interdisciplinarity is very useful... in facilitating the understanding of one's own topic from different points.

NA14: [Interdisciplinary research is] a good way of knowing what your discipline does. My own assumptions become clearer to me.

\section{Disadvantages}

Our respondents also emphasised some risks of interdisciplinary research: 


\section{It may lack sufficient rigour.}

For several respondents, scholars need to be trained within a well-contained field with its own standards and methodologies. Put simply, their concern was that too much pressure for interdisciplinary research causes scholars to become amateurs. In some responses the solution seemed to be to let scholars master their discipline before they reach out to others.

This point was made by 19 respondents, As6, As10, Au4, E2, E9, E14, LA2, LA4, ME3, ME4, NA1, NA3, NA4, NA14, NA16, R5 and:

R1: I think that the most important disadvantage of the interdisciplinary research is that there is a risk of remaining an amateur and missing some important nuances of some of the disciplines involved.

E8: There is an increasing threat to monodisciplinary research. People need to be deeply trained within a single discipline; there is a need for rigour. In the past rigour was exclusive, e.g. in Celtic studies medievalism was considered top of the tree. But there is a need to have intensive monodisciplinary training and then branch out.

2. Interdisciplinary work can be extremely time-consuming, starting from the basic information exchange needed at the outset, and then involving possibly years of hard work, often for an uncertain outcome.

This point was made by 7 respondents, As8, E1, E10, E12, E14, NA8, NA15, R9 and:

Af3: [interdisciplinary work] is difficult to carry out because it brings much greater complexity. It also requires that people from different disciplines work together and this isn't always easy. The advantage of monodisciplinary work by contrast is that it is much easier to carry out, involving a single perspective on an issue.

It is important to consider these objections in the light of distinctions between different ways of being interdisciplinary. If what is at issue is interdisciplinarity in the strong sense of A3 above, the first criticism has considerable force. But it has much less force against A1 and A2. Multidisciplinarity seems to get round the objection that the whole point is to allow scholars to remain masters in their field while still working with other experts from other fields on a collaborative project. However, 
this form of research is still vulnerable to the second criticism that the work involved is not that of mastering a new field, but of nurturing contacts, applying for project funds and running such projects, as well as having sufficient familiarity with the other disciplines to make the collaboration work, as in the interdisciplinarity of types A1 and A2.

\section{Institutional tensions}

These points help to set the stage for the key issue we wish to take up. There is no doubt that senior academic administrators and funding bodies often set a high premium on interdisciplinary research. In many countries and regions, funding schemes prioritise interdisciplinary and collaborative research. Some of the most prominent examples include the Mellon Foundation (USA), HERA (Humanities in the European Research Area), the Volkswagen Foundation, the AHRC (UK) and the NWO (Humanities) (the Netherlands). ${ }^{9}$

Turning to our interview respondents, we can present some extracts that make a version of this point:

As8: [Where is the push for interdisciplinary research coming from?] When applying for funding there have been new categories created which encourage people from different disciplines to come together and use interdisciplinary methods and skills. There is always a category which encourages this.

As10: Administrators do encourage interdisciplinary work. The reason perhaps is that they want to encourage important breakthroughs, which they think will come from interdisciplinary work. Perhaps the model comes from the natural sciences.

Af12: [What themes have been dominating your own field?] Most [history research in Nigeria] in recent times has become interdisciplinary, veering into engagements with the social sciences mostly. Attempts to engage with the mainstream of funding [e.g. to access available funding] have encouraged many emerging historians to veer into the social sciences.

E6: My own research is monodisciplinary and will probably remain so until I retire... But as a dean I strongly promote interdisciplinary research. Monodisciplinary research will surely be important also in the future, but for the humanities as a field it is important to engage with other disciplines, both within the humanities and with fields outside our own.... This presupposes cooperation with people from other fields. I think this development is under 
way. An example, when the [National] Research Council posted funding of new centres of excellence in 2011, four applications were sent from my faculty. All of them were interdisciplinary, involving researchers from other faculties. Five and ten years ago, this was not the case.

E11: There is, if there ever was, no longer a bright line with the humanities and the social sciences. There are many examples of interdisciplinary collaborations across that divide and many with the sciences and medical sciences, medical humanities for example, or areas where landscape architecture interfaces with educational sciences and engineering. They are too numerous to mention, but they tend to be the areas which, anecdotally speaking, seem to attract the most funding, because that really is what the big funders are interested in now.

Af2: Interdisciplinary research tends to be pushed by funders. Where there is strong university leadership, it tends to focus on attacking important social questions.

Af7: My own research has tended towards interdisciplinarity because research that is monodisciplinary is not attracting funding.

On the other hand, some interviewees reported a strong resistance to interdisciplinarity among researchers (perhaps mainstream academics?):

Af1: [Do you think this view on the benefits of interdisciplinarity is shared within ... your networks?] An emphatic NO, NO! Things around here tend to be trenchantly old school. You have your nice little cubicle.... You stay, and don't stray, in the cubicle.... I am an outlier - or better, a loose cannon - who broke down the walls of my cubicle. Still receiving flack though, because other members of the Cubicle (note the capitalisation!) are scratching their heads wondering why I have to leave the nice Cubicle.

E4: There is currently a lack of interdisciplinary engagement between my field and the social sciences. I was trained to respect the present, not like most humanities researchers, who revere the past. The social sciences also respect the present, but they study it by pursuing an obsession with quantitative data.... The general lack of interdisciplinary energy is the biggest problem for European universities. Until we change, we'll be inward looking, self-replicating, medieval corporations who pretend that we're socially relevant.

As7: The worst thing I suffered over the past ten years is the inertia of each and every discipline. In economics a professor does not need 
to communicate with a political scientist because this won't help them get published in the SCID journal. Even within the discipline of economics, those who are doing macroeconomics don't necessarily need to communicate with microeconomics. This is just no good. This is the number one challenge. This is the reason why I hold this interdisciplinary conference. It is very difficult, especially with Chinese academics. This type of interdisciplinary inertia is very strong. I've been striving to hold this ocean of inertia and I don't want to drown in it! [Also, from answer to publication question: They have no choice but to conduct research on smaller areas within disciplines.]

R6: For Russian humanities, though, [interdisciplinary] approaches are still novel, largely due to the fact that most researchers are locked into discipline-specific departments.

Some respondents went a step further and said that monodisciplinary attitudes manifest themselves in institutional ways:

NA7: The disadvantage for doing interdisciplinary work, especially for young scholars, is that professional life is still very much embedded within disciplines. And so you don't get credit for multi-authored publications, for example.

NA10: There are many challenges of interdisciplinary work. Many of the professional rewards are within disciplines and departments. Interdisciplinary work can be easily marginalised...

R9 (an environmental historian): Many disadvantages concern the position of an interdisciplinary researcher to find her/his place in the professional community. You are not a biologist any more, but for a long time even after getting a degree you are not considered to be a professional historian. It happens because you have no connections, you do not have a prominent teacher in your main field, thus you are not belonging to any group of disciples. This is particularly important for the scientific community in my country where many people have their own professional genealogy, being a disciple of a disciple of a famous intellectual.

If these sets of remarks are representative, there is likely to be a tension between funding priorities and more traditional attitudes and, indeed, we did find some respondents reporting such a tension:

NA5: Deans are very interested in interdisciplinary research. Deans want to be cutting edge, and that increasingly means 
interdisciplinary research. Often [they are] trying to recruit people whose academic and intellectual home is somewhat split between two fields. Generally, we think that's exciting and a good thing, but it also means that it's a challenge for universities and departments when it comes to evaluation and promotion. This is increasingly problematic and becoming more acute.... My conception of interdisciplinarity is that it is one person whose work is addressed to multiple communities. When that is the case, it may be hard for those communities to feel like the person is fully theirs. Sometimes the closest fields have the most difficulty agreeing on standards of excellence and what is authorship. That can be dangerous for junior faculty. [It] requires that junior faculty must be very proactive, making sure they are communicating with their groups [and] must have a disciplinary home, a core discipline. If you veer too far from home, that can be dangerous.

E5: On the one hand, the interdisciplinary trend is, in some parts of the competitive stream, encouraged; that's the ERC trend, the European Science Foundation trend (essentially the ERC trend) and also in some parts of national funding. For example, our government in Denmark strongly encourages interdisciplinary research and, in a recent evaluation of the research council system that was published a few years ago, it was one of the major recommendations. So that's one trend. You have goals that prioritise interdisciplinary research and on the other hand you have very strong gearing of governing publications that have a very good track record, have a very high gate impact factor, and so forth. And that sort of pushes research towards overspecialisation.

E14: Some will promote interdisciplinary research.... But research is mainly monodisciplinary in our humanities faculty although politics and the rectorat tend to encourage interdisciplinary research.

The basic issue is that the institutional incentives may be working in contradictory directions. When it comes to individual hiring and promotion, publishing monodisciplinary books and journals is paramount. But, at the same time, some funding bodies and senior academic administrators are promoting interdisciplinary research. This tension can manifest itself in a number of ways.

\section{Career advancement:}

There is a possible tension between the promotion of interdisciplinary research projects and publication regimes. As we have seen, a scholar's 
publication record is key to their hiring and promotion (and tenure, where applicable). The more prestigious the outlet, the more useful it is for these purposes. The issue is, are the most prestigious journals monodisciplinary? To settle this question definitively one would have to conduct a wide-ranging survey of humanities journals. The purpose of this chapter is to raise the question, and to suggest some anecdotal evidence, that monodisciplinarity does put a scholar at an advantage in terms of career advancement (through publication).

The problem would affect researchers at different stages of their career:

Early career: consider someone who has been recruited as a postdoctoral researcher on a funded interdisciplinary project. Their PhD has been monodisciplinary, but being a postdoc on such a project is their only (or best) career option at the next stage. Since they will have to publish in interdisciplinary formats as part of the project they will not be well placed at the end of the period to apply for permanent academic positions if the greatest prestige still goes to peer-reviewed papers in monodisciplinary journals.

Tenure: take a scheme where a university actually establishes interdisciplinary tenure-track positions (there are some initiatives of this nature in the US, for instance). The search committee will typically consist of members from different departments who will have to agree on a single candidate with good interdisciplinary credentials. At least from the hiring point of view this is not a handicap, but an advantage. But as one looks ahead to promotion and tenure, problems loom. Typically, the position will be located in an individual department. When it comes to the tenure decision, the department will decide according to the usual criteria. Here again, there is a risk that the bias towards monodisciplinary publications will put the candidate at a severe disadvantage.

Senior researchers: the incentive to publish monodisciplinary research will also deter well-established scholars from engaging in interdisciplinary research. Their promotion to more senior levels, such as named chairs, could be set back by the time taken putting together funding proposals and seeing them through.

An additional problem, which affects all stages of academic promotion, concerns letters of recommendation. Promotion depends on strong referees and readers (whether chosen by the candidate or not), but there could be a problem if the more trusted and respected readers are 
themselves monodisciplinary researchers. When such a scholar writes a promotion report on a candidate who has done interdisciplinary work, they might disclaim knowledge of some aspects of the research ('it's not really my field'), and thereby sound lukewarm.

Multidisciplinary edited books:

A related problem is that funded research projects of this kind are typically expected to produce multi-authored interdisciplinary books. But who exactly will read them? If most scholars remain in their disciplinary silos, they may look on the papers in such volumes with disdain. The papers are written to be understood by people outside the field, and so won't deserve the respect they would have if written for specialists. ${ }^{10}$

\section{Conclusion}

There is a fundamental question to be asked: what is the source of the institutional pressure for interdisciplinary research? Is it based on sound intellectual reasons, or do institutional leaders think that the humanities should imitate the natural sciences (where interdisciplinary research is much more common)?

E5:... [the enthusiasm for interdisciplinarity] is not founded on any kind of solid evidence, it's founded here on suspicion or presuppositions that interdisciplinarity is good. I haven't seen robust or empirical evidence that this is the case. But on the other hand, I haven't seen robust evidence that delivering very narrow specialisation produces better research results. I suppose that at the end it's very difficult to get robust data on one or the other.

E13: As a general point, I don't think interdisciplinarity should be seen as good for its own sake.

NA1: I do think that interdisciplinary has become a buzzword. Sometimes it really lacks a certain heft.

On the other hand, one can also ask why the most prestigious publication outlets are monodisciplinary (if they are)? Is this merely a historical contingent fact?

There needs to be further discussion about the merits and best practices of interdisciplinary, comparative and multilingual research. Beyond that there needs to be a review of the underlying institutional incentives for research, and those who promote interdisciplinarity need to do more to use their influence to reform appointment and promotion procedures. 
Though this is already an acute problem in Europe and North America, there is every indication it will affect other regions as well.

Finally, we would like to state a little more explicitly where we ourselves stand on the nature and value of interdisciplinary research. We have seen how our respondents discussed its advantages under several headings. One of these was that many research questions simply cannot be addressed within an existing discipline; they require us to trespass across existing boundaries and create interdisciplinary projects. We strongly agree. Disciplinary boundaries should not be allowed to impose constraints on the kinds of research questions we pursue, and we should not be put off asking a question because its answer lies in a discipline other than the one we work in. It is the research question that should have primacy and the academic system, including institutional arrangements and individual incentives, should be set up to reflect this.

It would take us too far afield to examine the nature of interdisciplinary research in any detail, especially the well-known question about the contingency of disciplinary boundaries and the extent to which they are the result of historical circumstances and whether they fashion reality at its natural joins? But prima facie, it is not difficult to see how important a role contingency plays in the formation of disciplines. One can easily imagine how some disciplines (or sub-disciplines) arose because an individual researcher, or group of researchers, became curious about a set of questions. If these researchers have the appropriate entrepreneurial skills they might manage to draw other people's attention to their activities and succeed in institutionalising their curiosity. As soon as this happens a discipline has been established. Once in place, the discipline will attract the interest of people who consider their interests to be very similar to the now institutionalised curiosity of those who founded the discipline. Professional expertise evolves. But then someone may appear whose own intellectual curiosity does not fit within the existing discipline; they pose broader questions than it is able to answer, and so trespass across its boundaries to draw in knowledge from other disciplines. If this researcher shares similar entrepreneurial talents with their forerunners, they too will institutionalise their curiosity, and their interdisciplinary activities will become the foundation of a new discipline. So the process goes on and, at any stage, a discipline may seem (or be made to seem) as if it is simply natural, despite the fact that its development involved a significant level of contingency.

This is only a sketch. There have been a myriad processes by which different disciplines and sub-disciplines have been formed but the model is useful for thinking about disciplinary boundaries. The main point it 
brings out, and the point several of our own respondents made, is the primacy of the research question over whatever disciplinary boundaries happen to exist at any one time. Often, new research questions are imposed from outside academia, for instance, as a way of meeting large societal challenges (as in environmental studies, which demand the pooling of intellectual resources from history, philosophy and many of the social and natural sciences).

At any rate, for those who endorse the priority of research question over existing disciplinary boundaries, it is all the more important to face up to institutional barriers to interdisciplinary research.

(c) (i) Except where otherwise noted, this work is licensed under a Creative Commons Attribution 3.0 Unported License. To view a copy of this license, visit http://creativecommons.org/licenses/by/3.0/ 